\title{
Original article \\ The diseases we cause: Iatrogenic illness in a department of internal medicine
}

\author{
Sofia Madeira $^{\mathrm{a}, *, 1}$, Miguel Melo ${ }^{\mathrm{b}}$, João Porto ${ }^{\mathrm{c}}$, Sílvia Monteiro ${ }^{\mathrm{d}}$, J.M. Pereira de Moura ${ }^{\mathrm{e}}$, \\ M.B. Alexandrino ${ }^{\mathrm{e}}$, J.J. Alves Moura ${ }^{\mathrm{e}}$ \\ ${ }^{a}$ Department of Internal Medicine - Medicina 2, University Hospital (HUC), Praceta Mota Pinto, 3000 Coimbra, Portugal \\ ${ }^{\mathrm{b}}$ Department of Endocrinology, University Hospital (HUC) l, Coimbra, Portugal \\ ${ }^{c}$ Department of Internal Medicine - Medicina 2, University Hospital (HUC), Coimbra, Portugal \\ ${ }^{\mathrm{d}}$ Department of Cardiology, University Hospital (HUC)l, Coimbra, Portugal \\ ${ }^{\mathrm{e}}$ Department of Internal Medicine - Medicina 2, University Hospital (HUC), Coimbra, Portugal
}

Received 3 April 2006; received in revised form 7 November 2006; accepted 15 December 2006

\begin{abstract}
Background: The aim of this study was to estimate the incidence, main causes, and risk factors of iatrogenic disease occurring in a department of internal medicine.

Methods: Over a 1-year period, physicians systematically filled out a 2-page questionnaire for all patients admitted to the ward. A database was created and the data were statistically analyzed. Patients undergoing immunosuppressive, chemo-, or radiation therapy were excluded. Missing data were completed by reviewing the patients' charts. The patients were then divided into two groups: those with and those without iatrogenic disease. The groups were compared using several parameters including gender, age, social features, days of hospitalization, associated illness, functional status, medical impression, prognosis, associated renal or liver function impairment, drugs taken daily, and outcome. In the group with iatrogenic disease, the type, severity, and predictability were also analyzed.

Results: Of the 879 patients admitted to the ward, 445 completed questionnaires and were included in the study. A total of 102 patients (22.9\%) developed 121 iatrogenic events. Forty-four patients (43.1\%) were admitted for iatrogenic illness, 10 (9.8\%) developed lifethreatening events, and in $3(6.8 \%)$ it was the cause of death. Fifty-eight patients $(56.8 \%)$ registered 77 episodes of iatrogenic disease during their hospital stay, 20 (19.6\%) developed life-threatening events, and $9(11.7 \%)$ died, $4(5.2 \%)$ of an iatrogenic cause (nosocomial infections). Significant differences were found in 20 out of 26 parameters studied ( $p<0.005$ for all cases; $95 \%$ confidence interval). Eighteen percent of all iatrogenic disease was severe, $61.9 \%$ predictable, $54.5 \%$ avoidable, and $59 \%$ drug-related, $80 \%$ of which was due to side effects or adverse reactions. Infection and metabolic and electrolyte disorders were the most frequent effects.

Conclusions: It is possible to identify risk factors for iatrogenic events. Chronically ill elderly inpatients are the main target of iatrogenic events.
\end{abstract}

(C) 2007 European Federation of Internal Medicine. Published by Elsevier B.V. All rights reserved.

Keywords: Iatrogenic disease; Incidence; Internal medicine department; Prospective study; Risk factors

\footnotetext{
* Corresponding author. Tel.: +351 239400426.

E-mail addresses: amadeira@huc.min-saude.pt, madeirasofia@gmail.com (S. Madeira).

${ }^{1}$ R. Padre Américo, 42, 3ํ dto. 3000-313 Coimbra, Portugal. Tel.: +351 912248422 .
}

\section{Introduction}

From the Greek words iatrós, meaning "medical", and geneá, meaning "origin", iatrogenic means the occurrence of negative effects caused by a medical procedure. It is frequently thought that iatrogenic means "error" or "neglect", but iatrogenic effects and medical errors are opposite terms, not synonymous ones [1]. An error is a 
mistake or the result of ignorance and, thus, opposes the concept of medical attitude, whereas an iatrogenic effect is the consequence of an accurate action based on a correct indication and adequate criteria and can be predicted by the physician [1]. When, in trying to heal, relieve, or treat a patient, the physician (like any other health care worker) generates psychological, functional, or organic illness that takes the from of pain, disease, or disturbance, he is being iatrogenic. The diagnosis may be difficult, delayed, or initially missed as iatrogenic illness can be generated directly from the doctor-patient relationship or by means of agents used in the diagnostic search, or as a consequence of a therapeutic, instrumental (technical), or drug-related measure $[1,2]$.

Iatrogenic disease is a serious problem with a great social impact. It occurs very frequently, is expensive [3-8,12-16], and is potentially responsible for high morbidity and mortality $[5,6,8,12-14,17,18]$. In the U.S., it is estimated that iatrogenic causes are responsible for 225,000 deaths each year, thus being the third leading cause of death after heart disease and cancer [5]. Iatrogenic events have a high incidence $[10-12,19]$; some studies show that $3.7 \%[8,9]$ to $17 \%[4,20]$ of all patients admitted to the hospital experience some kind of iatrogenic event. In three major prospective studies carried out in 1980, 1986, and 1993 in different departments of internal medicine with 815, 1176, and 1549 patients each, the incidence found was 36\% [12], 25.1\% [19], and $14.7 \%$ [10], respectively. More recent studies show that iatrogenic events occur in $14-25 \%[10,19]$ of hospitalized patients in internal medicine departments and in $33 \%$ of patients over 65 years of age [18]. Almost onethird of the patients have iatrogenic disease before hospital admission [20] and 3-7\% of all admissions have iatrogenic causes $[10,15,16,21,22]$, increasing to almost $8 \%$ in patients over 65 years of age [11]. Iatrogenic events are also an important cause of admission to intensive care units (ICUs) [23-27] and of hospital readmission [28,29]. Little epidemiological data is available $[6,10]$. However, the few studies that are available have shown that there are some important predictors for the occurrence of iatrogenic events, namely, older age $[10,11,14,18-21]$, longer hospital stay $[5,10$, $15,18-21]$, number of drugs taken daily [11,15,18,21,32], associated pathologies [21], poor general medical status on admission [19], associated renal function impairment or failure [18], and IV catheterization [19]. Amongst the many types of iatrogenic diseases, the most common seem to be drugrelated $[6,9,10,12,16,21,23,30-33]$ or adverse drug reactions (ADRs). ADRs are frequent, costly and, in some cases, responsible for severe complications [6]. Generally, the patients do not know the reasons, details, or side effects of their medical treatment [21]. Depending on the series, the drugs most often referred to are anti-hypertensive drugs (mainly diuretics), cardiovascular drugs (mainly digoxin and digitalics; up to $15 \%$ ), NSAIDs (up to $12 \%$ ), hypoglycemic agents (6-12\%), anti-coagulants (10-11\%), antibiotics (Abs; approximately $7 \%$ ), and neuropsychiatric drugs $[10,11,14,19,21,23]$. The most frequent alterations found are serum electrolyte disturbances (including dehydration), metabolic/endocrine disorders, gastrointestinal and liver disorders, cardiovascular disorders, neuropsychiatric events, hematological disorders, and infectious and hemorrhagic complications $[10,11,15,17-$ 21]. According to the definition of type A or B (Table 1), in order to be avoidable, an ADR must be predictable. Older patients tend to suffer more type A reactions, whereas type B reactions seem to occur more often in younger patients $[2,23]$. In the published studies, some $16-50 \%$ of all ADRs are serious $[12,14,18-20,30]$ and $22-80 \%$ avoidable/preventable $[6,9,10,16,20-22]$. Some authors suggest strategies to fight this "pandemic", including enhanced educational programs from the pre-graduate to post-graduate level, aimed at prescribing physicians, patients, the general public, the pharmaceutical industry, health authorities, epidemiologists, and medical educators; measures of risk reduction (e.g., decreasing hospital stay, number of drugs, and technical procedures); the setting up of databases or electronic systems to diminish errors and ADRs; and determined efforts to improve ambulatory care $[6,12-14,16,17,19,20,23,34]$.

There are several definitions of iatrogenic disease (or iatrogenic illness, pathology, effect, reaction, or event). The authors have chosen one that is broad enough to include nosocomial infection, a major cause of mortality and morbidity amongst the inpatient population [35] and even after discharge [3]. The incidence of nosocomial infection in hospitalized patients in internal medicine wards can be as high as $2.6 \%[35,36]$, the most frequent infections observed being urinary tract infections (UTIs), pneumonias, Clostridium difficille-associated diarrhea, wound infections, and sepsis [8,19,35-37]. These infectious complications are usually associated with old age, longer hospital stay, poor general medical status on admission, IV catheterization, mechanical ventilation, urinary catheterization, and female sex $[19,35]$. Thus, iatrogenic disease is defined as any medical, therapeutic, diagnostic, or prophylactic action that, unintentionally, causes symptoms that need treatment, call for hospital admission, increase hospital stay, cause permanent incapacity or injury, or lead to death. Overall, departments of

Table 1

Types of adverse drug reactions (ADRs)

Averse drug - An event that is noxious and unintended and occurs reaction with doses used in humans for prophylaxis, diagnosis, therapy, or modification of physiological functions (intentional or deliberate overdose or drug abuse are excluded)

Type A reaction - Caused by known toxicity of the drug, dose-related and pharmacological effect (like bleeding caused by warfarin)

- Potentially preventable

Type B reaction - Idiosyncratic or allergic in nature

- Reactions that usually occur from the initial use of a drug in a patient are not predictable and, therefore, not preventable 
Table 2

Iatrogenic events before and after admission and related mortality

\begin{tabular}{lrr}
\hline Iatrogenic disease episodes (121) & $N$ & $\%$ \\
\hline Patients admitted for iatrogenic disease & 44 & 36.36 \\
Lead to patients' death & 5 & 11.36 \\
Due to iatrogenic disease & 3 & 6.82 \\
Events occurring in the ward (58 inpatients) & 77 & 63.64 \\
Lead to patients' death & 9 & 15.51 \\
Due to iatrogenic disease (nosocomial infections) & 4 & 6.89 \\
\hline
\end{tabular}

internal medicine are, and will be, at the forefront of this problem [16].

The aim of this prospective study was to identify, quantify, and characterize episodes and types of iatrogenic disease occurring in hospitalized patients in a department of internal medicine (Medicina 2-HUC) over a 12-month period (from March 1, 2003 to March 1, 2004). We also sought to shed light on risk factors for the occurrence of iatrogenic disease in our inpatients and, thus, suggest the necessary strategies to prevent or avoid them.

\section{Materials and methods}

This was a prospective study done by inviting all inpatients in the department to systematically complete a 2-page questionnaire, showing electronic prescriptions, over the course of 1 year. Permission was obtained from the ethics committee. Every questionnaire answered was later reviewed. For every incomplete questionnaire, discharge reports and clinical files were reviewed, allowing missing data to be completed. Data underwent statistical analysis and results were reported.

The study was carried out by seven physicians in the department, four of whom were responsible in the ward (two created the database and carried out the statistical analysis) and three senior advisers. The responsible physicians filled out the questionnaires and the senior physicians independently reviewed them; they then decided by consensus which cases were due to iatrogenic episodes. For those suffering from iatrogenic disease, the disease was described and classified by severity and type. The senior physicians then further classified the iatrogenic events by predictability, avoidability, and type.

Patients undergoing cytostatic (QT), immunosuppressive (IS), or radiation therapy (RT) were excluded, as were patients who stayed less than 2 days (short hospital stay), those with no electronic drug prescriptions, and "social cases". The latter refers to patients who stay in the hospital after clinical discharge for several reasons (e.g., lack of cooperation from next-of-kin, no fixed residence, abandonment by family, loss of vacancy in nursing home, etc.) These

Table 3

General characteristics of the study population and comparison of the two groups

\begin{tabular}{|c|c|c|c|c|c|c|c|c|c|c|c|}
\hline \multirow{2}{*}{$\frac{\text { Study population }}{\text { Data analyzed }}$} & & \multicolumn{2}{|c|}{ Iatrogenic disease } & \multicolumn{4}{|c|}{ Chi-square } & \multicolumn{4}{|c|}{ Strength of association } \\
\hline & & With & Without & $\chi^{2} ; d f$ & $P$ value & $\begin{array}{l}P \text { value } \\
\text { summary }\end{array}$ & Significant & RR & $95 \% \mathrm{CI}$ & OR & $95 \% \mathrm{CI}$ \\
\hline \multirow[t]{2}{*}{ Gender } & 우 & $46(45.1 \%)$ & $186(54.22 \%)$ & $2.626 ; 1$ & 0.105 & ns & $\mathrm{N}$ & 0.7542 & $0.535-1.063$ & 0.6934 & $0.4447-1.081$ \\
\hline & ত & $56(54.9 \%)$ & $157(45.78 \%)$ & & & & & & & & \\
\hline Age & $\begin{array}{l}\text { Years }^{\mathrm{a}} \\
(\text { mean } \pm \mathrm{SD})\end{array}$ & $77.56 \pm 12.8$ & $70.77 \pm 18.28$ & $5.609 ; 1$ & 0.0179 & $*$ & $\mathrm{Y}$ & 1.875 & $1.075-3.269$ & 2.171 & $1.129-4.174$ \\
\hline \multirow[t]{3}{*}{ Educational level } & Illiterate & 36 & 86 & & & & & & & & \\
\hline & Literate; & 58 & 211 & $4.128 ; 1$ & 0.0422 & $*$ & $\mathrm{Y}$ & 1.444 & $1.019-2.047$ & 1.63 & $1.015-2.618$ \\
\hline & $\begin{array}{l}\text { Higher } \\
\text { education }\end{array}$ & 8 & 46 & & & & & & & & \\
\hline \multirow[t]{3}{*}{ Origin } & Home & 64 & 271 & & & & & & & & \\
\hline & $\begin{array}{l}\text { Nursing } \\
\text { home }\end{array}$ & 23 & 37 & $11.65 ; 1$ & 0.0006 & $* * *$ & $\mathrm{Y}$ & 1.83 & $1.305-2.567$ & 2.275 & $1.409-3.672$ \\
\hline & Hospital & 15 & 34 & & & & & & & & \\
\hline \multirow[t]{3}{*}{ Admission } & Urgent & 92 & 289 & & & & & & & & \\
\hline & Elective & 10 & 54 & $2.447 ; 1$ & 0.1177 & ns & $\mathrm{N}$ & 1.574 & $0.8659-2.86$ & 1.757 & $0.8606-3.587$ \\
\hline & None & 59 & 208 & & & & & & & & \\
\hline $\begin{array}{l}\text { Previous hospital } \\
\text { stay }\end{array}$ & $\begin{array}{l}>1 \text { in the last } \\
12 \text { months }\end{array}$ & 43 & 135 & $0.313 ; 1$ & 0.645 & ns & $\mathrm{N}$ & 1.104 & $0.782-1.557$ & 1.137 & $0.7255-1.781$ \\
\hline Hospital stay & $\begin{array}{l}\text { Days }^{b} \\
(\text { mean } \pm \text { SD) }\end{array}$ & $13.52 \pm 8.465$ & $9.09 \pm 5.797$ & $5.727 ; 1$ & 0.0167 & $*$ & $\mathrm{Y}$ & 1.986 & $1.084-3.639$ & 2.317 & $1.146-4.685$ \\
\hline \multirow{2}{*}{$\begin{array}{l}\text { Associated } \\
\text { chronic condition }\end{array}$} & None & 9 & 76 & $9.362 ; 1$ & 0.0022 & $* *$ & $\mathrm{Y}$ & 2.475 & $1.302-4.707$ & 2.991 & $1.442-6.206$ \\
\hline & Yes & 93 & 267 & & & & & & & & \\
\hline
\end{tabular}

우: female sex; $\sigma^{\urcorner}$: male sex; $\chi^{2}$; df: chi-square difference; $P$ value summary: ${ }^{*}$ minimum to $* * *$ maximum significance; n.s.: not significant; significant: statistically significant in two-tailed test with alpha $<0.05$; Y: yes; N: no; RR: relative risk; OR: odds ratio; CI: confidence interval; SD: standard deviation.

a Risk calculated for ages $>70$ years; $t$ student test applied to mean age: $p<0.0001$.

b Risk calculated for hospital stay $>4$ days; $t$ student test applied to mean hospital stay: $p<0.0001$. 
Table 4

Comparison of the two groups at admission

\begin{tabular}{|c|c|c|c|c|c|c|c|c|c|c|c|}
\hline \multirow{2}{*}{$\begin{array}{l}\text { At admission } \\
\text { Data analyzed }\end{array}$} & & \multicolumn{2}{|c|}{$\begin{array}{l}\text { Iatrogenic } \\
\text { disease }\end{array}$} & \multicolumn{4}{|c|}{ Chi-square } & \multicolumn{4}{|c|}{ Strength of association } \\
\hline & & With & Without & $\chi^{2} ; d f$ & $P$ value & $\begin{array}{l}P \text { value } \\
\text { summary }\end{array}$ & Significant & $\mathrm{RR}$ & $95 \% \mathrm{CI}$ & OR & $95 \% \mathrm{CI}$ \\
\hline \multirow[t]{3}{*}{ Prognosis } & Mild & 20 & 168 & & & & & & & & \\
\hline & Moderate & 47 & 118 & $26.61 ; 1$ & $<0.0001$ & $* * *$ & $\mathrm{Y}$ & 2.999 & $1.91-4.71$ & 3.936 & $2.31-6.706$ \\
\hline & Severe & 35 & 57 & & & & & & & & \\
\hline \multirow{3}{*}{$\begin{array}{l}\text { Global clinical } \\
\text { impression }\end{array}$} & Good & 15 & 145 & & & & & & & & \\
\hline & Average & 35 & 109 & $22.76 ; 1$ & $<0.0001$ & $* * *$ & $\mathrm{Y}$ & 2.242 & $1.607-3.128$ & 2.968 & $1.879-4.689$ \\
\hline & Bad & 52 & 89 & & & & & & & & \\
\hline \multirow{2}{*}{$\begin{array}{c}\text { Functional } \\
\text { status }\end{array}$} & Not dependent & 47 & 97 & $11.80 ; 1$ & 0.0006 & $* * *$ & $\mathrm{Y}$ & 1.805 & $1.291-2.522$ & 2.199 & $1.395-3.466$ \\
\hline & Dependent & 55 & 246 & & & & & & & & \\
\hline \multirow{3}{*}{$\begin{array}{l}\text { Level of } \\
\text { awareness }\end{array}$} & Aware & 40 & 206 & & & & & & & & \\
\hline & Confused & 45 & 105 & $4.379 ; 1$ & 0.0377 & $*$ & $\mathrm{Y}$ & 1.616 & $1.054-2.48$ & 1.944 & $1.030-3.669$ \\
\hline & Coma & 17 & 32 & & & & & & & & \\
\hline $\begin{array}{l}\text { Associated renal } \\
\text { failure/ }\end{array}$ & No & 33 & 222 & $33.67 ; 1$ & $<0.0001$ & $* * *$ & $\mathrm{Y}$ & 2.806 & $1.938-4.062$ & 3.836 & $2.397-6.14$ \\
\hline $\begin{array}{l}\text { impaired renal } \\
\text { function }\end{array}$ & Yes & 69 & 121 & & & & & & & & \\
\hline $\begin{array}{l}\text { Associated hepatic } \\
\text { failure/ }\end{array}$ & No & 57 & 234 & $7.062 ; 1$ & 0.0079 & $* *$ & $\mathrm{Y}$ & 1.583 & $1.13-2.218$ & 1.835 & $1.169-2.88$ \\
\hline $\begin{array}{l}\text { impaired liver } \\
\text { function }\end{array}$ & Yes & 45 & 109 & & & & & & & & \\
\hline Drugs & $\begin{array}{l}\mathrm{Nr}^{\mathrm{a}} \\
(\text { mean } \pm \mathrm{SD})\end{array}$ & $\begin{array}{l}5.42 \pm \\
2.375\end{array}$ & $\begin{array}{l}3.472 \pm \\
2.44\end{array}$ & $20.59 ; 1$ & $<0.0001$ & $* * *$ & $\mathrm{Y}$ & 10.15 & $2.554-40.37$ & 13.38 & $3.216-55.63$ \\
\hline \multirow[t]{9}{*}{ Which? } & $\mathrm{ABs}$ & 5 & 14 & $1.799 ; 1$ & 0.1799 & ns & $\mathrm{N}$ & 1.376 & $0.877-2.158$ & 1.539 & $0.8166-2.899$ \\
\hline & ACEIs & 9 & 14 & $4.930 ; 1$ & 0.0264 & $*$ & $\mathrm{Y}$ & 1.484 & $1.052-2.092$ & 1.689 & $1.06-2.69$ \\
\hline & $\begin{array}{l}\text { Anti-platelet/ } \\
\text { anti-coagulants }\end{array}$ & 3 & 1 & $4.656 ; 1$ & 0.0309 & $*$ & $\mathrm{Y}$ & 1047 & $1.041-2.067$ & 1.669 & $1.045-2.665$ \\
\hline & Diuretics & 12 & 19 & $6.044 ; 1$ & 0.014 & $*$ & $\mathrm{Y}$ & 1.528 & $1.089-2.144$ & 1.744 & $1.116-2.725$ \\
\hline & $\begin{array}{l}\text { Digitalics/ } \\
\text { digoxin }\end{array}$ & 2 & 8 & $3.958 ; 1$ & 0.0466 & $*$ & $\mathrm{Y}$ & 1.508 & $1.021-2.228$ & 1.747 & $1.004-3.041$ \\
\hline & Insulin & 4 & 9 & $6.698 ; 1$ & 0.0097 & $* *$ & $\mathrm{Y}$ & 1.768 & $1.18-2.65$ & 2.225 & $1.20-4.125$ \\
\hline & $\begin{array}{l}\text { Anti-depressants/ } \\
\text { neuroleptics }\end{array}$ & 3 & 21 & $0.9785 ; 1$ & 0.3226 & ns & $\mathrm{N}$ & 1.193 & $0.8425-1.69$ & 1.26 & $0.796-1.993$ \\
\hline & Steroids & 2 & 9 & $0.1376 ; 1$ & 0.7107 & ns & $\mathrm{N}$ & 1.147 & $0.563-2.333$ & 1.199 & $0.4597-3.125$ \\
\hline & NSAIDs & 7 & 22 & $0.6946 ; 1$ & 0.4046 & ns & $\mathrm{N}$ & 1.209 & $0.78-1.873$ & 1.286 & $0.711-2.325$ \\
\hline
\end{tabular}

$\chi^{2} ; d f$ : chi-square difference; $P$ value summary: ${ }^{*}$ minimum to $* * *$ maximum significance; n.s.: not significant; significant: statistically significant in two-tailed test with alpha <0.05; Y: yes; N: no; RR: relative risk; OR: odds ratio; CI: confidence interval; SD: standard deviation; Nr: number; AB's: antibiotics; ACEI's: ACE inhibitors; NSAID's: non-steroid anti-inflammatory drugs.

a Risk calculated for $\mathrm{Nr}$ of drugs on admission $\geq 2 ; t$ student test applied to mean $\mathrm{Nr}$ of drugs on admission: $p<0.0001$.

patients were treated for their condition and included in the study until they were clinically ready for discharge; the remaining stay was excluded. Questionnaires with missing data that were impossible to complete, even after a review of clinical files (discharge reports and electronics chart), were also excluded.

The database was created in Excel and the statistical analysis was done using Prism4. The $\chi^{2}$ test was used for categorical variables (contingency tables) and Student's $t$-test for nonparametrical variables. All $P$ values, relative risk (RR) and odds ratio (OR) results were obtained with two-tailed tests for an alpha below $5 \%$ and a confidence interval of $95 \%$. The results are presented as a comparison of the two groups (those with iatrogenic illness versus those without).

\subsection{Questionnaire}

The information requested on the questionnaire included:

Identification: Name; sex; age; occupation; degree of education (higher education; literate; illiterate)

Origin: Hospital; home; nursing home

Type of admission: Elective; urgent; date of admission; date of discharge; previous hospitalizations in the last 12 months

Diagnosis (or hypothesis) and prognosis: Diagnosis on admission; diagnosis at discharge; prognosis (mild: no life expectancy reduction by sex and age group; moderate: life expectancy $>3$ years; severe: life expectancy $<3$ years) 
Table 5

Comparison of the two groups during in-hospital stay

\begin{tabular}{|c|c|c|c|c|c|c|c|c|c|c|c|}
\hline \multirow{2}{*}{\multicolumn{2}{|c|}{$\begin{array}{l}\text { During hospital stay } \\
\text { Data analyzed }\end{array}$}} & \multicolumn{2}{|c|}{ Iatrogenic disease } & \multicolumn{4}{|l|}{ Chi-square } & \multicolumn{4}{|c|}{ Strength of association } \\
\hline & & \multirow{2}{*}{$\begin{array}{l}\text { With } \\
7.373 \pm \\
2.261\end{array}$} & \multirow{2}{*}{$\begin{array}{l}\text { Without } \\
5.438 \pm \\
2.323\end{array}$} & \multirow{2}{*}{$\frac{\chi^{2} ; d f}{41.4 ; 1}$} & \multirow{2}{*}{$\frac{P \text { value }}{<0.0001}$} & \multirow{2}{*}{$\begin{array}{l}P \text { value summary } \\
* * *\end{array}$} & \multirow{2}{*}{$\begin{array}{l}\text { Significant } \\
\mathrm{Y}\end{array}$} & \multirow{2}{*}{$\frac{\mathrm{RR}}{3.08}$} & \multirow{2}{*}{$\frac{95 \% \mathrm{CI}}{2.139-4.437}$} & \multirow{2}{*}{$\frac{\mathrm{OR}}{4.415}$} & \multirow{2}{*}{$\frac{95 \% \mathrm{CI}}{2.756-7.074}$} \\
\hline Drugs & $\mathrm{Nr}^{\mathrm{a}}(\mathrm{mean} \pm \mathrm{SD})$ & & & & & & & & & & \\
\hline \multirow[t]{9}{*}{ Which? } & AB's & 21 & 48 & $0.0842 ; 1$ & 0.7717 & ns & $\mathrm{N}$ & 0.9439 & $0.6401-1.392$ & 0.9253 & $0.5477-1.563$ \\
\hline & ACEI's & 9 & 20 & $1.271 ; 1$ & 0.2595 & ns & $\mathrm{N}$ & 1.221 & $0.8647-1.724$ & 1.298 & $0.8241-2.045$ \\
\hline & $\begin{array}{l}\text { Anti-platelet/ } \\
\text { anti-coagulants }\end{array}$ & 1 & 2 & $7.224 ; 1$ & 0.0072 & $* *$ & Y & 1.661 & $1.134-2.433$ & 1.91 & $1.186-3.075$ \\
\hline & Diuretics & 20 & 28 & $0.03819 ; 1$ & 0.8451 & ns & $\mathrm{N}$ & 0.9623 & $0.655-1.413$ & 0.9455 & $0.539-1.659$ \\
\hline & $\begin{array}{l}\text { Digitalics/ } \\
\text { digoxin }\end{array}$ & 3 & 12 & $1.332 ; 1$ & 0.2485 & ns & $\mathrm{N}$ & 1.266 & $0.8546-1.876$ & 1.368 & $0.8025-2.33$ \\
\hline & Insulin & 10 & 14 & $3.391 ; 1$ & 0.0656 & ns & $\mathrm{N}$ & 1.414 & $0.9856-2.028$ & 1.587 & $0.9682-2.602$ \\
\hline & $\begin{array}{l}\text { Anti-depressants/ } \\
\text { neuroleptics }\end{array}$ & 5 & 13 & $1.041 ; 1$ & 0.3076 & ns & $\mathrm{N}$ & 1.21 & $0.8421-1.74$ & 1.285 & $0.7931-2.082$ \\
\hline & Steroids & 9 & 13 & $0.04108 ; 1$ & 0.8394 & ns & $\mathrm{N}$ & 1.05 & $0.6585-1.673$ & 1.065 & $0.5782-1.963$ \\
\hline & NSAID's & 1 & 6 & $0.3271 ; 1$ & 0.5674 & ns & $\mathrm{N}$ & 0.8394 & $0.4549-1.549$ & 0.8003 & $0.3725-1.719$ \\
\hline
\end{tabular}

$\chi^{2} ; d f$ : chi-square difference; $P$ value summary: $*$ minimum to $* * *$ maximum significance; n.s.: not significant; significant: statistically significant in two-tailed test with alpha <0.05; Y: yes; N: no; RR: relative risk; OR: odds ratio; CI: confidence interval; SD: standard deviation; Nr: number; AB's: antibiotics; ACEI's: ACE inhibitors; NSAID's: non-steroid anti-inflammatory drugs.

${ }^{a}$ Risk calculated for $\mathrm{Nr}$ of drugs during hospital stay $\geq 3 ; t$ student test applied to mean $\mathrm{Nr}$ of drugs during hospital stay: $p<0.0001$.

Clinical condition on admission (a): Global clinical impression (good, average; bad; dependent); level of awareness (A) coma; confused; conscious/aware); associated renal failure/impaired renal function (A); hepatic failure/impaired liver function (A); associated chronic illness (which?);

Clinical condition at discharge (d): Outcome (D) (better; same condition; worse; death; transferred for iatrogenic illness); level of awareness (D) (coma; confused; conscious/aware); hepatic failure/impaired liver function (D) (encephalopathy; ascites; jaundice; albumin; INR; prothrombin time); renal failure/impaired renal function (D) (creatinine; BUN)

Drug-taking history: Drugs on admission (previous 4 weeks); patient's knowledge about: i) the disease; ii) the medication; iii) the side effects; drugs during hospital stay; drugs at discharge

Iatrogenic illness: Yes, no; description; severity (mild: no need for specific treatment; moderate: caused or increased hospital stay or needed specific treatment; severe: caused incapacity or death); type (drugrelated; other)

Review by senior physicians

Iatrogenic disease: Yes; no: predictable; avoidable; drugrelated (inadequate doses; inappropriate indication; incorrect administration; drug interaction; side effects or adverse reactions); other (technical procedure; falls; diagnostic errors; pressure ulcers; dietetic errors)

After the first 2 months, preliminary results were presented to all of the physicians in the department and a set of objectives was established. The major events and those most often observed were reviewed. Since ours is a university hospital, trainees are included in the wards and perform some simple techniques (it was assumed they also play a role in iatrogenic events). Certain rules were implemented during the ongoing study in order to try to reduce some events, such as hematomas post-arterial catheterization by local compression for no less than 3 min, patient-to-patient contamination by judicious hand washing after every contact, efforts to reduce the number of drugs taken daily by the patients, and special attention to older patients.

\section{Results}

\subsection{Study population}

From March 1, 2003 to March 1, 2004, 879 patients were admitted to our department. One hundred sixty-four of these patients died, accounting for an $18.65 \%$ mortality rate. A total of 457 questionnaires (52\%) were answered, though only $266(58.2 \%)$ were complete; the rest needed to be completed by reviewing discharge reports and clinical files (electronics chart). The questionnaires of 12 patients (2.63\%) were excluded: 7 of whom were undergoing QT or RT, 1 "social case" (this patient had been discharged before the beginning of the study and was staying in the hospital for social reasons since there was a lack of cooperation from the family, who refused to take him home), and 4 with an insufficiently answered questionnaire (even after review of the clinical file, by accessing discharge reports and electronics charts, it was not possible to complete the missing data in these cases). A total of 445 patient questionnaires were ultimately included in the analysis. 
Table 6

Comparison of the two groups at discharge

\begin{tabular}{|c|c|c|c|c|c|c|c|c|c|c|c|}
\hline \multirow{2}{*}{$\begin{array}{l}\text { At discharge } \\
\text { Data analyzed }\end{array}$} & & \multicolumn{2}{|c|}{$\begin{array}{l}\text { Iatrogenic } \\
\text { disease }\end{array}$} & \multicolumn{4}{|c|}{ Chi-square } & \multicolumn{4}{|c|}{ Strength of association } \\
\hline & & With & Without & $\chi^{2} ; d f$ & $P$ value & $\begin{array}{l}P \text { value } \\
\text { summary }\end{array}$ & Significant & RR & $95 \% \mathrm{CI}$ & OR & $95 \% \mathrm{CI}$ \\
\hline \multirow{3}{*}{$\begin{array}{l}\text { Global clinical } \\
\text { impression }\end{array}$} & Good & 29 & 172 & $7.084 ; 1$ & 0.0078 & $* *$ & $\mathrm{Y}$ & 1.894 & $1.223-2.934$ & 2.444 & $1.245-4.798$ \\
\hline & Average & 43 & 112 & & & & & & & & \\
\hline & Bad & 16 & 26 & & & & & & & & \\
\hline \multirow[t]{2}{*}{ Functional status } & $\begin{array}{l}\text { Not } \\
\text { dependent }\end{array}$ & 20 & 54 & $2.654 ; 1$ & 0.1033 & ns & $\mathrm{N}$ & 1.404 & $0.9413-2.096$ & 1.563 & $0.9108-2.680$ \\
\hline & Dependent & 67 & 258 & & & & & & & & \\
\hline \multirow[t]{3}{*}{ Level of awareness } & Aware & 50 & 237 & $11.06 ; 1$ & 0.0009 & $* * *$ & $\mathrm{Y}$ & 1.873 & $1.3-2.698$ & 2.298 & $1.397-3.779$ \\
\hline & Confused & 37 & 72 & & & & & & & & \\
\hline & Coma & 0 & 3 & & & & & & & & \\
\hline $\begin{array}{l}\text { Associated renal } \\
\text { failure/ }\end{array}$ & No & 73 & 280 & $6.622 ; 1$ & 0.0101 & $*$ & $\mathrm{Y}$ & 1.668 & $1.141-2.44$ & 1.976 & $1.169-3.339$ \\
\hline $\begin{array}{l}\text { impaired renal } \\
\text { function }\end{array}$ & Yes & 29 & 63 & & & & & & & & \\
\hline \multicolumn{12}{|l|}{$\begin{array}{l}\text { Associated hepatic } \\
\text { failure/ }\end{array}$} \\
\hline \multirow{2}{*}{$\begin{array}{l}\text { impaired liver } \\
\text { function }\end{array}$} & No & 62 & 239 & $1.184 ; 1$ & 0.2766 & ns & $\mathrm{N}$ & 1.255 & $0.838-1.881$ & 1.344 & $0.788-2.293$ \\
\hline & Yes & 25 & 72 & & & & & & & & \\
\hline Drugs & $\begin{array}{l}\mathrm{Nr}^{\mathrm{a}} \\
(\text { mean } \pm \mathrm{SD})\end{array}$ & $\begin{array}{l}4.909 \pm \\
1.74\end{array}$ & $\begin{array}{l}4.236 \pm \\
2.249\end{array}$ & $12.79 ; 1$ & 0.0003 & $* * *$ & $\mathrm{Y}$ & 2.281 & $1.403-3.710$ & 2.792 & $1.566-4.977$ \\
\hline \multirow{3}{*}{$\begin{array}{l}\text { Patient's knowledge } \\
\text { about }\end{array}$} & Disease & 39 & 186 & $8.806 ; 1$ & 0.003 & $* *$ & $\mathrm{Y}$ & 0.5918 & $0.4156-0.8427$ & 0.5071 & $0.3225-0.7974$ \\
\hline & Medication & 30 & 147 & $5.933 ; 1$ & 0.0149 & $*$ & $\mathrm{Y}$ & 0.6309 & $0.4309-0.9238$ & 0.5556 & $0.3449-0.8949$ \\
\hline & Side effects & 11 & 85 & $9.104 ; 1$ & 0.0026 & $* *$ & $\mathrm{Y}$ & 0.4394 & $0.2452-0.7877$ & 0.3669 & $0.1874-0.7185$ \\
\hline \multirow[t]{5}{*}{ Outcome } & Better & 66 & 231 & $4.041 ; 1$ & 0.0444 & $*$ & $\mathrm{Y}$ & 1.525 & $1.027-2.263$ & 1.776 & $1.009-3.124$ \\
\hline & $\begin{array}{l}\text { Same } \\
\text { condition }\end{array}$ & 15 & 64 & & & & & & & & \\
\hline & Worse & 6 & 15 & & & & & & & & \\
\hline & Death & 15 & 31 & & & & & & & & \\
\hline & Mortality rate & $14.71 \%$ & $9.04 \%$ & 2.725 .1 & 0.0988 & ns & $\mathrm{N}$ & 1.496 & $0.9486-2.358$ & 1.735 & $0.8961-3.36$ \\
\hline
\end{tabular}

$\chi^{2} ; d f$ : chi-square difference; $P$ value summary: $*$ minimum to $* * *$ maximum significance; n.s.: not significant; significant: statistically significant in two-tailed test with alpha $<0.05$; Y: yes; N: no; RR: relative risk; OR: odds ratio; CI: confidence interval; SD: standard deviation; Nr: number.

${ }^{\text {a }}$ Risk calculated for $\mathrm{Nr}$ of drugs at discharge $\geq 4 ; t$ student test applied to mean $\mathrm{Nr}$ of drugs at discharge: $p=0.0054$.

In the series studied, the mortality rate was $10.33 \%$, and 102 inpatients registered 121 episodes of iatrogenic disease, yielding a $22.9 \%$ incidence. In 343 inpatients, there was no iatrogenic illness. Of all the patients suffering episodes of iatrogenic disease, $44(43.13 \%)$ were admitted for iatrogenic events; 5 of them (11.36\%) died, $3(6.82 \%)$ from the admission cause, so iatrogenic disease was considered to be the cause of death. In 58 inpatients, 77 episodes $(63.63 \%)$ of iatrogenic disease were registered in the ward, 4 of which $(6.89 \%)$ also lead to the patient's death, all due to nosocomial infections (Table 2). A total of $9(15.51 \%)$ out of 58 inpatients died during their hospital stay; iatrogenic events were thus fatal in nine cases. Iatrogenic events were fatal in seven cases, accounting for a mortality rate of $1.6 \%$ of all

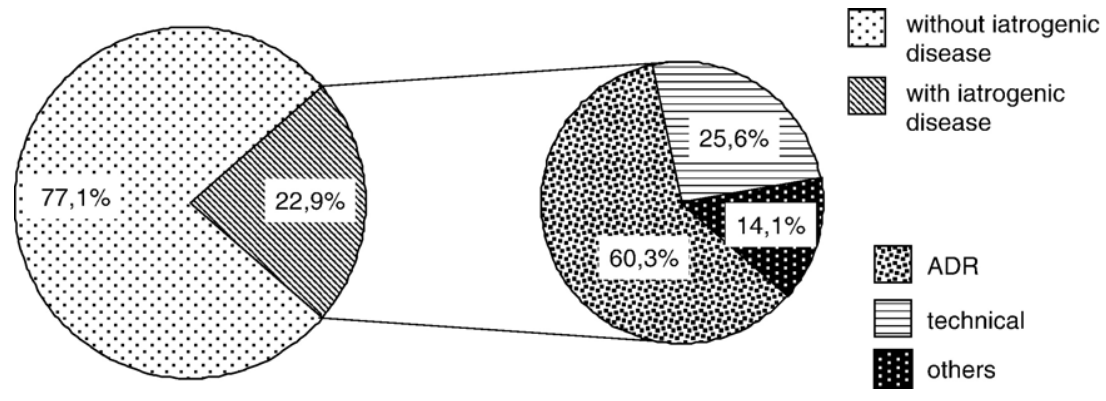

Fig. 1. Incidence and main causes of iatrogenic disease. 


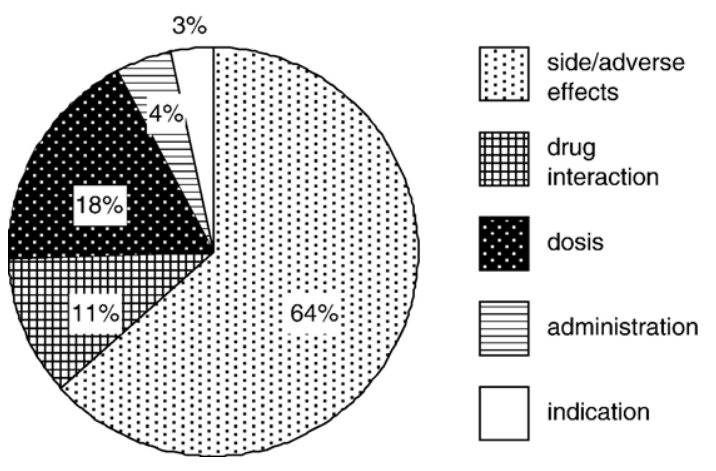

Fig. 2. Types of ADR's.

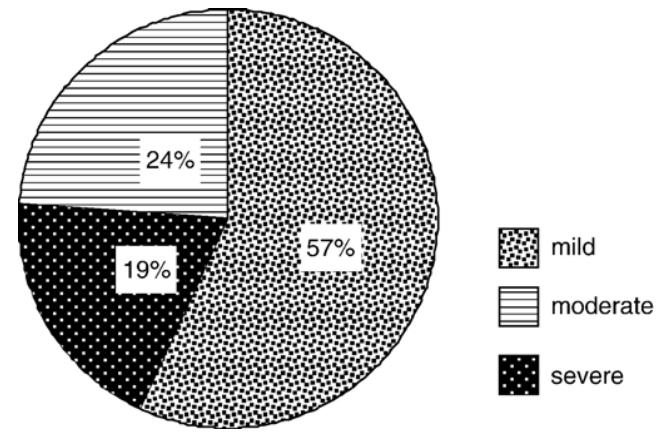

Fig. 4. Severity of iatrogenic disease. included admissions and $6.86 \%$ of all patients suffering from iatrogenic events.

The two groups were similar in male/female ratio. The group suffering from iatrogenic disease was, on average, 6.79 years older than the disease-free group, and over the age of 70, the risk of iatrogenic events increased significantly. The mean age in the first group was $77.56 \pm 12.8$ (range 14-101) years and in the second group $70.77 \pm 18.28$ (range 29-98) years. Patients with a lower educational level, those with any associated chronic disease, and those coming from other hospitals or a nursing home were more affected by iatrogenic events (Table 3). No differences were found in either group in the type of admission (urgent or elective) or admissions in the previous year ( $<$ or $>6$ months). The patients suffering from iatrogenic disease had a significantly longer hospital stay on average, 4.43 days longer- and a stay longer than 4 days is significant in the increase of risk for iatrogenic events. The mean hospital stay was $13.52 \pm 8.46$ (range $1-32$ ) days in the first group and $9.09 \pm 5.79$ (range 1-28) days in the second group (Table 3).

Prognosis, patients' general functional status, and levels of awareness on admission were worse in the group with iatrogenic illness. Although the criterion is subjective and variable depending on the physician, the patients' global clinical impression was consistent with the other criteria and was also worse in the same group. Iatrogenic illness was more frequent in patients suffering from impaired renal

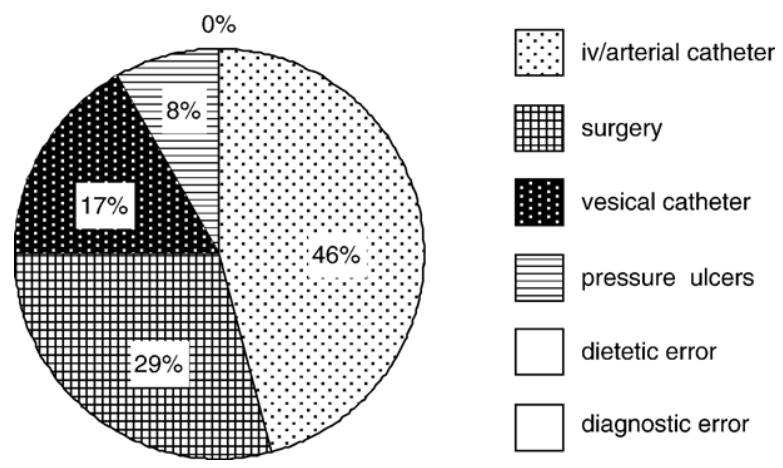

Fig. 3. Types of iatrogenic disease due to technical causes. function or renal failure on admission and in patients suffering from impaired hepatic function or liver failure on admission. On admission and in the previous 4 weeks, the group with iatrogenic events took more drugs daily -1.498 more - than the other group, and the patients taking 2 or more drugs showed a higher risk of iatrogenic events (Table 4). Diuretics, ACEIs, anti-coagulant/anti-platelet drugs, digitalics/digoxin, and insulin were significantly more frequent on admission in the patients who suffered from iatrogenic events (Table 4).

During hospitalization the occurrence of iatrogenic events was also found to be related to the number of drugs taken daily. The group with iatrogenic events was taking 1.93 more drugs than the other group, with the patients taking three or more drugs at the greatest risk (Table 5). Anti-coagulants/ anti-platelet drugs were more frequently prescribed to these patients during hospitalization (Table 5).

Both groups showed the same general functional status at discharge. Patients with a worse global clinical impression and patients with a lower level of awareness at discharge suffered more events of an iatrogenic illness. At discharge from the department, the group with iatrogenic illness was the one to show a higher incidence of impaired renal function or renal failure, yet, no significant differences were found between the two groups with relation to impaired hepatic function or liver failure at discharge. At discharge the number of iatrogenic events also proved to be related to the number of drugs prescribed, the group with iatrogenic illness taking 0.734 more drugs daily than the other group and the risk increasing for patients taking four or more drugs. The patients' knowledge about the disease, the medication, and the side effects was significantly lower in the group with iatrogenic illness, and this group also showed a worse clinical outcome. Both groups had a similar mortality rate (Table 6).

The incidence of iatrogenic illness was $22.9 \%$ in the series studied. Most iatrogenic events in this series were drug-related, followed by those resulting from technical procedures (Fig. 1). Some $60 \%$ of drug-related iatrogenic illnesses were due to side effects or ADRs, $20 \%$ to inappropriate doses, and $10 \%$ to drug interactions (Fig. 2). The technical causes of iatrogenic events were mainly 


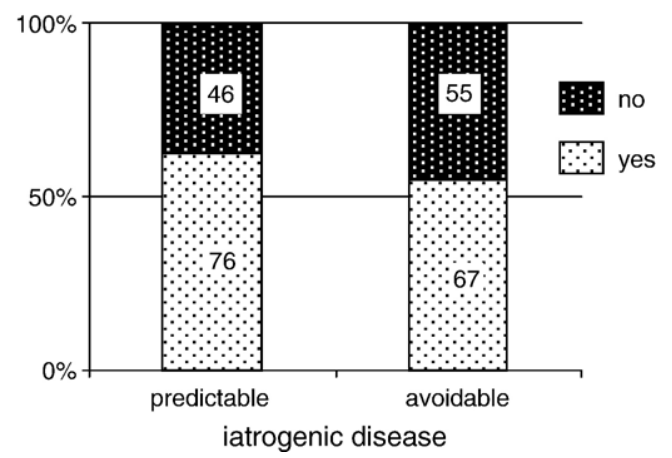

Fig. 5. Predictable and avoidable iatrogenic disease.

catheterization, either intravenous (IV), arterial, or urinary, followed by surgery and pressure ulcers (Fig. 3).

Infections were the most frequent disorders observed in this series $(24 \%)$, followed by electrolyte $(18 \%)$, metabolic/ endocrine $(12 \%)$, gastrointestinal $(8 \%)$, hematological/coagulation disorders $(9 \%)$, neurological $(4 \%)$, cardiovascular $(2.5 \%)$ and skin/allergic manifestations $(2 \%)$. There are records of nine respiratory infections, six urinary infections, two nosocomial conjunctivitis, one head trauma after a fall from bed, and one post-liver biopsy hematoma (patient did not abide to the physician's recommendation to stay in bed).

Of the confirmed events of iatrogenic illness, about $18 \%$ were classified as severe and $24 \%$ as moderate (Fig. 4), causing death in $2.02 \%$ of the study population $(8.82 \%$ in the group with iatrogenic illness).

As in series in several other studies, iatrogenic events proved to be $61.9 \%$ predictable and $54.55 \%$ avoidable (Fig. 5).

The risk factors identified by this study are: age over 70 years, lower educational level, transfer from other institutions, hospital stay longer than 4 days, associated chronic condition, severe prognosis on admission, global clinical impression on admission and at discharge, general functional status on admission, level of awareness on admission and at discharge, associated renal failure or impaired renal function on admission and at discharge, associated hepatic failure or impaired liver function on admission, number of drugs taken ( 2 or more drugs on admission, 3 or more drugs during hospital stay, 4 or more drugs at discharge), and the patients' knowledge about the disease, the medication, and its side effects. Iatrogenic disease can also be considered a risk factor for a worse outcome.

\section{Discussion}

The most difficult task to accomplish in the present study was to obtain the cooperation of all practitioners with regard to filling out the questionnaires, even though there was one responsible physician for each sector in the ward. In fact, during the summer months (from June to September) and in December, a much smaller number of questionnaires were filled out compared to during other periods of the year, these being the most common holiday periods.
Although the preliminary results (obtained after the first 2 months and presented to all physicians in the department) were not considered in this study, the overall comparison of the 2-month and 12-month results shows a slight reduction in iatrogenic events. The number of events may have been reduced after implementation of the simple rule that a review of the major events and those most often observed lead to.

Reading the results can create a false idea that the population studied has a lower mortality rate than the total population of inpatients (total of admissions); this is not accurate. Such results occur most probably because some patients died within the first $24 \mathrm{~h}$ after admission; thus, the questionnaires were not completed, regardless of the cause of death. In fact, in all completed questionnaires, less than $10 \%$ correspond to patients who died, but the real mortality rate is almost twice as high.

The incidence of iatrogenic events is high in the population studied as it includes nosocomial infection. Still, it may be underestimated, as only about half of the patients admitted were analyzed (only 51.88\% completed questionnaires).

Overall, the results found are very similar to those found in the published medical literature in terms of incidence, risk factors, severity, avoidability, and type of iatrogenic disease. The size of our study population was large enough for us to draw conclusions and to identify risk factors for iatrogenic events, and that is precisely what we did.

It is very important to increase patients' knowledge about their diseases and their medication and to inform them about possible side effects and ADRs as a way of reducing iatrogenic events. It also seems fair to conclude that all iatrogenic events should be reviewed and used for comprehensive management, as was attempted by presenting the preliminary results. Through a common strategy, established objectives, and teamwork, it is possible to forecast and to change attitudes and behavior to "avoid the avoidable".

Electronic prescription may be helpful for physicians as it can help reduce errors, provide information on drug safety, calculate doses, and alert them to possible drug interactions.

Finally, and as redundant as it may seem, we have once again shown that elderly patients with chronic disease and polypharmacy are the preferential "targets" of iatrogenic illness. They should receive increased attention from all health care workers.

\section{Learning points}

- Iatrogenic events have a high incidence in internal medicine wards. They are mainly drug-related (ADRs), due to nosocomial infections or to technical, diagnostic, and therapeutic procedures.

- As expected, older patients, patients with a longer hospital stay, those who take several drugs daily, and those who exhibit serious conditions (that can worsen the prognosis) have a much higher incidence of iatrogenic disease. The physician's judgement of the patient's clinical impression is an important risk predictor. 
- Iatrogenic events may worsen the patients' outcome, as an isolated risk factor.

- It is possible to avoid many of the diseases we cause since most of them are predictable and avoidable. It is important to pay special attention to high-risk groups.

\section{Acknowledgments}

The authors would like to acknowledge all of the students, practitioners, residents, and specialists in the department whose help made this study possible.

\section{References}

[1] Cerecedo Cortina Vicente B. Iatrogenia y error medico. Rev Med Hosp Gen Mex 1997;60(2):75-83.

[2] Alhalel Gabay B. Polifarmacia, Iatrogenia y Reacciones Adversas por Medicamentos en el Adulto Mayor. Diagnóstico: Revista Médica de la Fundación Instituto Hipólito Unanue, vol. 42(3); May-Jun 2003. p. 117-21.

[3] Forster A, Clark H, Menard A, Dupuis N, Chernish R, Chandok N, et al. Adverse events among medical patients after discharge from hospital. CMAJ Feb 3 2004;170(3):345-9.

[4] Starfield B. Is US really the best in the world? JAMA Jul 26 2000;284 (4):483-5

[5] Zhan C, Miller M. Excess length of stay, charges and mortality attributable to medical injuries during hospitalization. JAMA Oct 8 2003;290(14):1868-74.

[6] Kaushal R, Bates D, Landrigan C, McKenna K, Clapp M, Frederico F, et al. Medication errors and adverse drug events in paediatric inpatients. JAMA Apr 25 2001;285(16):2114-20.

[7] Diez Jarilla J, Munoz Bellido J, de Castro del Pozo S. Iatrogenic pathology in an internal medicine service-I: global and non-drug iatrogenic pathology. Med Clin (Barc) Jun 7 1986;87(2):58-61.

[8] Brennan T, Leape L, Laird N, Hebert L, Localio A, Lawthers A, et al. Incidence of adverse events and negligence in hospitalized patients: results of the Harvard medical practice study I. NEJM Feb 7 1991;324 (6):370-6.

[9] Leape L, Brennan T, Laird N, Lawthers A, Localio A, Barnes B, et al. The nature of adverse events in hospitalized patients: results of the Harvard medical practice study II. NEJM Feb 7 1991;324(6):377-84.

[10] Sampereiz Legarre A, Rubio Obanos M, Escolar Castellon F, Alonso Martinez J, Lanas Arbeloa A, Ayuso Blanco T. A iatrogenic pathology study in an internal medicine service. Rev Clin Esp Jun 1994;194 (6):457-63.

[11] Fradet G, Legac X, Charlois T, Ponge T, Cottin S. Iatrogenic druginduced diseases, requiring hospitalization, in patients over 65 years of age. 1-year retrospective study in an internal medicine department. Rev Med Interne 1996;17(6):456-60.

[12] Steel K, Gertman P, Crescenzi C, Anderson J. Iatrogenic illness on a general medical service at a university hospital. NEJM Mar 12 1981;304(11):638-42.

[13] Weingart S, Iezzoni L. Looking for medical injuries where the light is bright. JAMA Oct 8 2003;290(14):1917-9.

[14] Queneau P, Chabot J, Rajaona H, Boissier C, Grandmottet P. Iatrogenic illness observed in the hospital environment-I: a report of 109 cases collected in a cross-sectional APNET study. Bull Acad Natl Med Apr 1992;176(4):511-26 discussion 526-9.

[15] Moore N, Lecointre D, Noblet C, Mabille M. Frequency and cost of serious adverse drug reactions in a department of general medicine. Br J Clin Pharmacol Mar 1998;45(3):301-8.

[16] Lagnaoui R, Moore N, Fach J, Longy-Boursier M, Begaud B. Adverse drug reactions in a department of systemic diseases-oriented internal medicine: prevalence, incidence, direct costs and avoidability. Eur J Clin Pharmacol May 2000;56(2):181-6.

[17] Gurwitz J, Field T, Harrold L, Rothschild J, Debellis K, Seger A, et al. Incidence of adverse drug events among older persons in the ambulatory setting. JAMA 2003;289: 1107-16.

[18] Gonzales-Martin G, Yanez L, Valenzuela F. Adverse drug reactions among hospitalized elderly patients: prospective study. Rev Med Chil Oct 1997;125(10):1129-36.

[19] de la Sierra A, Cardellach F, Cobo E, Bove A, Roige M, Santos M, et al. Iatrogenic illness in a department of general internal medicine: a prospective study. Mt Sinai J Med Sep 1989;56(4):267-71.

[20] Husi F, Stalder H. Self-monitoring of physicians for the detection of iatrogenic diseases. Schweiz Med Wochenschr Mar 15 1986;116 (11):346-51.

[21] Lepori V, Perren A, Marone G. Adverse internal medicine drug effects at hospital admission. Schweiz Med Wochenschr Jun 19 1999;129 (24):915-22.

[22] Lakshmanan M, Hershey C, Breslau D. Hospital admissions caused by iatrogenic disease. Arch Int Med Oct 1 1986;146(10).

[23] Wiffen P, Gill M, Edwards J, Moore E. Adverse drug reactions in hospital patients: a systematic review of the prospective and retrospective studies. Bandolier extra; Jun 2002.

[24] Trunet P, Le Gall J, Lhoste F, Regnier B, Saillard Y, Carlet J, et al. The role of iatrogenic disease in admissions to intensive care. JAMA Dec 12 1980;244(23).

[25] Lee CT, Guo HR, Chen JB. Hyponatremia in the emergency department. Am J Emerg Med May 2000;18(3):264-8.

[26] Rothschild J, Landrigan C, Cronin J, Kaushal R, Lockley S, Burdick E, et al. The critical care safety study: the incidence and nature of adverse events and serious medical errors in intensive care. Crit Care Med Aug 2005;33(8):1694-700 comment 1860-2.

[27] Bouhaja B, Mermech M, Mestiri T, Ben Ayed M, Dhaouadi M, Ben Ammar M. Iatrogenic disease in the intensive care unit: a prospective study. Reanim Urgences Jul 1999;8(4):319-26.

[28] Alonso Martinez JL, Llorante Diez B, Echegaray Agara M, Urbieta Echezarreta MA, Gonzalez Arencibia C. Hospital readmission in internal medicine. An Med Interna May 2001;18(5):248-54.

[29] Dormann H, Neubert A, Criegee-Rieck M, Egger T, Radespiel-Troger M, Azaz-Livshits $\mathrm{T}$, et al. Readmissions and adverse drug reactions in internal medicine: the economic impact. J Intern Med Jun 2004;255 (6):653-63.

[30] Lazarou J, Pomeranz B, Corey P. Incidence of Adverse Drug Reactions in hospitalized patients. JAMA Apr 15 1998;279(15):1200-5.

[31] Runciman W, Webb R, Helps S, Thomas E, Sexton E, Studdert D, et al. A comparison of iatrogenic injury studies in Australia and the USA. II: Reviewer behavior and quality of care. Int J Qual Health Care Oct 2000;12(5):379-88.

[32] Roblot P, de Bayser L, Barrier J, Marechaud R, Becq-Giraudon B. Primum non nocere, a forgotten adage at times. Apropos of 106 cases of iatrogenic pathologies collected in a year. Rev Med Interne 1993;14(10):950.

[33] Goute D, Gonon N, Roblot P, Marechaud R, Sudre Y. Iatrogenic drug pathology on admission in a department of internal medicine; results of a 6-month prospective study. Ann Med Interne (Paris) 1987;138 (2):148-9.

[34] McGavock H. Prescription-related illness: a scandalous pandemic. J Eval Clin Pract Nov 2004;10(4):491-7.

[35] Zamir D, Polychuck I, Leibovitz I, Reiblat T, Zamir C, Scharf S. Nosocomial infections in internal medicine departments. Harefuah Apr 2003;142(4):265-8.

[36] Munar Ques M, Font Gelabert A, Feliu Mazaira L, Mut Mandilego A, Vidal Mullor R, Vich Martorell C, et al. Nosocomial diseases in a general medicine service. Med Clin (Barc) Nov 1987;89(15):625-30.

[37] Riedinger J, Robbins L. Prevention of iatrogenic illness: adverse drug reactions and nosocomial infections in hospitalized older adults. Clin Geriatr Med Nov 1998;14(4):681-98. 